\title{
Identification of a $\beta$-amyloid-binding plasma protein, LRP1: implications for biomarker research and therapy in Alzheimer's disease
}

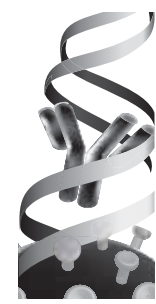

\author{
HenrikZetterberg \\ Göteborg U niversity, \\ Institute of N euroscience \& \\ Physiology, D epartment of \\ Psychiatry \& N eurochemistry, \\ The Sahlgrenska Academy, \\ S-431 80 M ölndal, Sweden \\ Tel.: +46 313432 377; \\ Fax: +46 313432 426; \\ E-mail: henrik.zetterberg@ \\ gu.se
}

\begin{abstract}
Evaluation of Sagare A, Deane R, Bell RD et al.: Clearance of amyloid- $\beta$ by circulating lipoprotein receptors. Nat. Med. (2007) (Epub ahead of print) [1]. Sagare and coworkers have identified a major amyloid- $\beta(A \beta)$-binding protein in plasma low-density lipoprotein receptor-related protein-1 (LRP1). This protein may bind 70-90\% of the $A \beta$ that circulates in peripheral blood and seems to function as a peripheral sink for Alzheimer's disease (AD) causing brain $A \beta$. Using a mouse model of $A D$, the authors demonstrate that boosting the capacity of the sink by administering a recombinant form of soluble LRP1 (SLRP1) reduces brain amyloid plaque load and improves learning and memory. They extend these results by demonstrating that patients with AD have depressed plasma SLRP1 levels. Their data suggest that exogenously administered, recombinant SLRP1 may provide a novel approach to reduce or reverse the AD-related build up of $A \beta$ in the brain. Furthermore, it would be interesting to explore the possibility that reduced SLRP1 levels in plasma may be a specific biochemical sign of incipient AD.
\end{abstract}

Amyloid- $\beta$ (A $\beta$ ) clearance may be of profound importance in the pathogenesis of sporadic Alzhe imer's disease (AD) [1], which is the most common form of dementia, accounting for $50-60 \%$ of approximately 20-30 million dementia cases around the world [2]. Clinical signs of $A D$ are characterized by progressive cognitive deterioration, together with neuropsychiatric symptoms or behavioral changes. The pathological hallmarks of $A D$ are the presence of intracellular neurofibrillary tangles of hyperphosphorylated protein tau and extracellular deposits of $A \beta$-protein in senile plaques in the cerebral cortex. Although these brain lesions may also be observed in aged nondemented individuals, the accumulation of $A \beta$ in the brain is believed by many to represent the earliest event in the disease process [2].

$A \beta$ is generated from amyloid precursor protein, a membrane-spanning protein expressed by most cells in the body, by enzymatic digestion involving $\beta$ - and $\gamma$-secretase activities. Once formed, $A \beta$ can undergo degradation by the many proteases that are present in the brain interstitial fluid [3], stabilization in monomeric forms by binding to cerebrospinal fluid (CSF) apolipoprotein $E$ and other chaperonelike amyloid-binding proteins, such as $\beta$-trace protein [4], or aggregation to synaptotoxic oligomers. A $\beta$ may also be cleared across the blood-brain barrier to the blood by low-density lipoprotein receptor-related protein-1 (LRP1) [5].

The amyloid cascade hypothesis holds that an imbalance between the production and clearance of $A \beta$ is the primary alteration in the $A D$ process [6]. Although still somewhat controversial, the hypothesis has survived many attempts of falsification and has al ready translated into a series of advanced therapeutic approaches that are in different stages of clinical trial. The main focuses are to inhibit brain $A \beta$ production (e.g., by the use of $\beta$ - or $\gamma$-secretase inhibitors and inhibitors of amyloid precursor protein mRN A translation) and aggregation (e.g., by the use of $A \beta$ oligomerization inhibitors), and to increase $A \beta$ clearance from the brain (e.g., by active or passive $A \beta$ immunotherapy) [7]. These types of drugs are likely to have the best efficacy in the early or even preclinical stage of the disease, when the synaptic and neuronal loss has not become too widespread. Therefore, there is a great need for biomarkers that identify AD preclinically, or in the mild cognitive impairment stage of the disease.

O ne of the most promising biomarkers for $A D$ is the $A \beta$ peptide per se. A wealth of evidence shows that reduced levels of the 42 amino acid form of $A \beta, A \beta 42$, in CSF is a reliable biochemical sign of $A D$, especially when interpreted together with CSF levels of tau proteins [3]. H owever, despite the known safety of lumbar punctures [8], many researchers and clinicians are trying to find AD biomarkers in peripheral blood.

Several studies have examined plasma levels of $A \beta$ in $A D$ but the findings are contradictory. Some groups report high concentrations of either $A \beta 42$ or $A \beta 40$ in plasma of $A D$ patients, although with a broad overlap between patients 
and controls, whereas most groups find no change [9]. Apart from disease-related factors, the opposing data may be due to the fact that $A \beta 42$ is notoriously difficult to measure in plasma. The peptide is very hydrophobic and binds not only to certain test tube walls, but also to several plasma proteins, including albumin [10] and cystatin C [11].

\section{Results}

In a very recent and potentially groundbreaking investigation, Sagare and coworkers present data that may be of relevance both to the treatment and diagnosis of AD in the future [1]. They show that, in addition to mediating $A \beta$ transport across the blood-brain barrier, a soluble circulating variant of LRP1 (SLRP1) functions as a major $A \beta$-binding protein in peripheral blood. As much as $70-90 \%$ of total $A \beta$ in plasma may be bound to this protein. This may explain the phenomenon, known by many $A D$ biomarker researchers but rarely publicized, that addition of increasing amounts of detergent to their plasma $A \beta$ assays will increase the signal up to a certain threshold value when the antibody affinity is lost. In addition, Sagare and colleagues show that AD patients have approximately 30\% lower SLRP1 levels in plasma [1]. Finally, they show that administration of a recombinant form of SLRP1 to amyloid precursor protein-transgenic mice diminished amyloid plaque pathology in brain tissue and improved their memory. The treatment removed both vascular and parenchymal amyloid, and increased plasma $A \beta$, approximately half of which was associated with the LRP 1 analogue.

\section{Significance of the results \& future perspective}

$A D$ is a very common disease against which there is still no effective therapy. The result that peripheral SLRP1 can mobilize neurotoxic $A \beta$ from the brain in a transgenic mouse model of $A D$ lends support to the view that passive immunization using infusion of anti-A $\beta$ antibodies in the peripheral blood in humans may work in a similar manner. However, given the fact that there are more than 40 different treatments that cure transgenic $A D$ mice but have little or no effect in humans [2], the next important step will be to test the therapeutic potential of recombinant SLRP1 administration in humans. Furthermore, it will be worthwhile to test the possibility that a reduction in plasma SLRP1 may be an early and specific biochemical sign of incipient $A D$.

Financial \& competing interests disclosure Work in the author's group is supported by the Swedish Research Coundil, the Swedish Coundil for Working Life and Social Reearch, the EU project dNEUPRO and the Alzheimer Foundation. Theauthor hasno other relevant affiliations or financial involvement with any organization or entity with a financial interest in or financial conflict with the subject matter or materials discussed in the manuscript apart from thosedisclosed.

No writing assistance was utilized in the production of this manuscript.

\section{Bibliography}

1. Sagare A, D eane R, Bell RD et al.: C learance of amyloid- $\beta$ by circulating lipoprotein receptors. N at. M ed. (2007) (Epub ahead of print).

2. Blennow $\mathrm{K}$, de Leon MJ, Zetterberg $\mathrm{H}$ : Alzheimer's disease. Lancet 368(9533), 387-403 (2006).

3. Andreasson U, Portelius E, Andersson M E, Blennow $\mathrm{K}$, Z etterberg $\mathrm{H}$ : Aspects of $\beta$-amyloid as a biomarker for Alzheimer's disease. Biomarkers M ed. 1(1), 59-78 (2007).

4. Kanekiyo T, Ban T, AritakeK et al.: Lipocalintype prostaglandin $D$ synthase/ $\beta$-trace is a major amyloid $\beta$-chaperone in human cerebrospinal fluid. Proc. N atl Acad. Sci. USA 104(15), 6412-6417 (2007).
5. Shibata M, Yamada S, Kumar SR et al.: Clearance of Alzheimer's amyloid-ss(1-40) peptide from brain by $L D L$ receptor-related protein-1 at the blood-brain barrier. J. Clin. Invest. 106(12), 1489-1499 (2000).

6. H ardy J, Selkoe DJ: Theamyloid hypothesis of Alzheimer's disease: progress and problems on the road to therapeutics. Science 297(5580), 353-356 (2002).

7. Roberson ED, M ucke L: 100 years and counting: prospects for defeating Alzheimer's disease. Science 314(5800), 781-784 (2006).

8. Peskind ER, Riekse R, Q uinn JF et al.: Safety and acceptability of the research lumbar puncture. Alzheimer Dis. Assoc. Disord. 19(4), 220-225 (2005).
9. Irizarry M C: Biomarkers of Alzheimer disease in plasma. NeuroRx 1(2), 226-234 (2004).

10. Kuo YM, Emmerling M R, Lampert $H C$ et al.: $H$ igh levels of circulating $A \beta 42$ are sequestered by plasma proteins in Alzheimer's disease. Biochem. Biophys. Res. Commun. 257(3), 787-791 (1999).

11. Selenica M L, Wang $X, O$ stergaardPedersen $L$, Westlind-D anielsson $A$, Grubb A: Cystatin $C$ reduces the in vitro formation of soluble $A \beta 1-42$ oligomers and protofibrils. Scand. J. Clin. Lab. Invest. 67(2), 179-190 (2007). 